\title{
Mathematical modeling in drug discovery and development
}

\author{
R. Miftahof ${ }^{1} \&$ N. Akhmadeev ${ }^{2}$ \\ ${ }^{I}$ Pohang University of Science and Technology, Rep. of Korea \\ ${ }^{2}$ Kazan Medical University, Tatarstan, Russia
}

\begin{abstract}
Mathematical modeling and computer simulations are emerging technologies in drug discovery, development and drug assessment for short- and long-term biological effects. They promise to be cheap, practical, sensitive and specific, and capture real aspects of the disease phenotype that is accepted as clinically and biologically meaningful. However, existing computer-based platforms are not capable of integrating complex nonlinear interactions of molecular, cellular, tissue, organ and system activities that are required in drug discovery. Novel software, ABS Technologies $\odot$, is designed on a "reductionism-integration" principle. It offers solutions with regard to rational decision making at different stages of drug development. Examples of numerical investigations into the effects of Lotronex (GSK) and benzodiazepines provided valuable information related to the dynamics of visceral perception and motility of the gut. The results revealed the intricate role of nonlinear interplay between different signaling pathways in the pathogenesis of visceral hypersensitivity and functional gastrointestinal disorders. Advantages and limitations of the use of computer-based technologies in drug discovery are briefly reviewed.

Keywords: mathematical modeling, computer simulation, drug discovery.
\end{abstract}

\section{Introduction}

During the last decade the pharmaceutical industry has followed a simplistic assumption that a single drug hitting a single target was the "rational" way to design drugs. That was a direct consequence of our lack of knowledge about function-determining features of desired ligand molecules in the drug discovery process. Although this approach continues to dominate pharmaceutical thinking, 
there is an urgent need to change this paradigm. With recent advances in science and biotechnology - the development of receptor theory [1], the formulation of pharmaco-metabonomic phenotyping concept to personalizing drug treatment [2], the sequencing and mapping of the human genome [3], advances in combinatorial chemistry and computer-based de novo design of drug-like molecules [4-6], we may expect rapid and effective solutions to vital problems related to drug discovery and development - rational target identification and validation, effective assessment of pharmacokinetic properties of hit and lead compounds, pharmacodynamics of ligand-receptor interactions, ADMET (absorption, distribution, metabolism, excretion and toxicity) drug properties and prediction of short and long-term clinical effects. In contrast, it has been argued that the quality of target validation has deteriorated [7], although animal models of toxicity have performed fairly well, animal disease models have been poor approximators of human disease and, as a result, multiple high profile clinical trial failures in drug development have been reported, e.g., the anti-CD28 antibody (TGN1412) (TeGenero) for leukaemia caused a "cytokine storm" during a small Phase I trial, an anticoagulant drug - Exantra (ximelagatran) (AstraZeneca), was withdrawn in Phase III trials because of its liver toxicity, late-stage trials were halted on Pargluva (muraglitazar) (Bristol-Myers Squibb) the dual peroxosome proliferators-activated receptor agonist to treat diabetes type II, and torcetrapib (Pfizer) - a high-density lipoprotein cholesterol-raising drug, after they demonstrated increased cardiovascular risks [8].

The fundamental flaw of a single-target strategy is that it ignores the fact that the pathogenesis of disease, even caused by single genetic abnormality, is multifactorial and involves a sophisticated network system of unbalanced genes, proteins and signaling pathways. By targeting a typified factor for a given disease with a single-target drug one may achieve in vivo and in vitro short-term clinical effects. However, many existing drugs interact with more than one target. Many pathogenic targets have their multiple physiological functions and can interfere nonlinearly with various pathways. Therefore, the net result in most cases cannot be extrapolated from single effects and are difficult to access experimentally.

Drug development requires constant dynamic data integration and consolidation from systems biology at every stage of the arduous, lengthy (it takes $12-15$ years for a drug to reach the market) and expensive (at estimated cost of US\$ 1 billion) process. Mathematical modeling and computational implementations are at the heart of this effort. The advantages and limitations, successes and failures, support and critique, examples of emerging technology have been reviewed [6,9-11]. The aim of this paper is to illustrate the application of a novel computational platform, ABS Technologies $\odot$, (Advanced BioSimulations Technologies, Llc, USA) to unravel the neurochemical bases of visceral hypersensitivity and associated functional gastrointestinal disorder - a disabling condition known as the irritable bowel syndrome and to provide an "analytical" assessment of co-expressed chemical targets and receptor polymodality in terms of their interrelated mechanisms of action and possible relevance to therapy of disease and drug interactions. The study involved 
mathematical concepts and methodology proposed previously [12-14]. A brief description of the simulation setup is provided below.

\section{Description of the mathematical model}

\subsection{Physiological remarks}

Visceral hypersensitivity is a common finding in patients with functional gastrointestinal disorders and the irritable bowel syndrome, in particular. Although some progress has been made in unraveling the neurochemical pathophysiology of the disease, the exact neuropharmacology of the hypersensitivity remains illusive. The pivotal role in the receiving and processing of sensory information in the gut belongs to the primary sensory and motor neurons of the afferent neuronal pathway of the myenteric nervous plexus along with the afferent fibers of the principal ganglionic neuron. They have also been implicated in the formation of a space map for coordinated motility patterns including the generation of migrating myoelectrical complexes and active forces of contraction. Complex neurochemical processes of signal transduction, including the co-localization of multiple neurotransmitters at the presynaptic level and the expression of multiple classes of receptors at the post-synaptic membrane, add enormous plasticity to the system, allowing individual neurons to adapt quickly to constantly changing demands.

Acetylcholine $(\mathrm{ACh})$ is a major neurotransmitter in the enteric nervous system and the gut per se. Its effects are mediated by nicotinic and muscarinic receptors. The nACh receptors are non-selective ionotropic receptors, whereas $\mu \mathrm{ACh}$ receptors are part of the metabotropic - $\mathrm{G}$ - protein coupled receptors family. Activation of $\mathrm{nACh}$ receptors results in the generation of an inward calcium current with the production of fast or slow excitatory postsynaptic potentials, respectively. In contrast to the diverse effects of serotonin in the gastrointestinal tract, ACh always has an excitatory effect.

The ubiquitous biogenic amine 5-hydroxytryptamine (5-HT, serotonin) is present in the neurons of the enteric nervous system and to a greater extent in enterochromaffin cells. A distinct 5- $\mathrm{HT}_{3}$ receptor belongs to the family of ligand -gated ion channels. Serotonin applied by ionophoresis to a neuron with $5-\mathrm{HT}_{3}$ receptors causes a short latency $(<100 \mathrm{~ms})$ and a duration depolarization by invoking fast inward excitatory calcium, potassium and sodium currents. The 5$\mathrm{HT}_{4}$ receptors belong to a $\mathrm{G}_{\alpha \mathrm{s}}$ - protein - coupled family. The biological effect of their pharmacological activation is correlated with an increase in permeability of calcium-activated potassium and sodium channels and a decrease in permeability of potassium channels. The 5-HT - and ACh-receptors are expressed on the somas and presynaptic nerve terminals of the primary sensory and motor neurons and smooth muscle syncytia.

Two experimental setups were designed: 1) a one-dimensional model, that is composed of interconnected smooth muscle cells, the primary sensory and motor neurons, and 2) a spatio-temporal model of a functional unit of the gut, that incorporates a segment of the gut controlled by the myenteric nervous plexus 
[13]. The models replicate accurately the anatomical, histomorphological and intercellular topology. The one-dimensional model mimics the following sequence of events: i) deformation of the free nerve endings of the mechanoreceptors; ii) generation of action potentials and their propagation towards the soma of the primary neuron; iii) propagation of the wave of depolarization towards the soma of the motor neuron; iv) electrochemical coupling at the axodendritic synapse on the soma of the motor neuron and generation of the fast excitatory postsynaptic potential; v) discharge of the soma of the motor neuron and the propagation of action potentials towards the smooth muscle syncytium; vi) electrochemical coupling at the neuro-muscular synapse; vii) depolarization of the smooth muscle membrane and contraction-relaxation of smooth muscle.

In case of the spatio-temporal model, a segment of the gut with an enclosed solid non-deformable pellet was studied. The following chain of events were reproduced: i) discharge of a pacemaker cell; ii) initiation excitatory signals and their propagation within the myenteric nervous plexus; iii) electrochemical coupling at the neuro-muscular synapses on the smooth muscle syncytia; iv) production of active forces of contraction and propulsion of the bolus.

In both cases the deformation of smooth muscle syncytia led to excitation of the mechanoreceptors and release of serotonin from the enterochromaffin cells. With each experiment the effects of 5-HT and ACh alone, and as a result of their interactions through the process of co-activation of different receptor types, were analyzed. The effect of a 5- $\mathrm{HT}_{3}$ receptor antagonist - Lotronex (GSK) was achieved by varying conductances for affected channels. All numerical results presented below were obtained with ABS Technologies $\mathbb{C}$.

\section{Numerical examples}

\subsection{Effects of Lotronex (GSK)}

\subsubsection{Mechanoreceptors}

The mechanical deformation of the mechanoreceptors, caused the release of 5HT from enterochromaffin cells. Activation of the 5- $\mathrm{HT}_{3}$ receptors on the free nerve endings with a subsequent increase in permeability of ligand-gated ion channels caused an increase in the amplitude of action potentials to $26 \mathrm{mV}$. Serotonin reduced the threshold for mechanical activation of the mechanoreceptors by $17.6 \%$. Treatment of the mechanoreceptors with Lotronex (GSK) abolished the potassium current and significantly reduced the sodium influx. As a result, the dendritic action potentials of amplitude $3 \mathrm{mV}$ were generated. They were not strong enough to set off electrical signals and sustain their propagation.

\subsubsection{Primary sensory neuron}

The dendritic action potentials elicited at the free nerve endings propagated along the unmyelinated axon and reached the soma of the primary neuron. A selective stimulation of the $5-\mathrm{HT}_{3}$ receptors by $5-\mathrm{HT}$ had a profound effect on activity of all ion channels. The primary sensory neuron generated action potentials of $111.3 \mathrm{mV}$. Lotronex completely abolishes electrical activity of the 
neuron. However, the large deformation was able to induce action potentials of short duration of amplitude $91 \mathrm{mV}$. A gradual increase in the concentration of the antagonist dose-dependently reduced the excitability of neuron.

A concomitant stimulation of the co-localized 5- $\mathrm{HT}_{4}$ receptors resulted in an increase in excitability of the neuron. The dynamics of the sodium and potassium channels exhibited a high frequency oscillatory mode while the calcium current demonstrated a beating mode of activity. The neuron generated spikes of high amplitude $50 \mathrm{mV}$.

\subsubsection{Motor neuron}

Selective stimulation of 5- $\mathrm{HT}_{3}$ receptors by the free endogenous 5-HT in absence of ACh did not have excitatory effect on the soma of the motor neuron. Co-joint excitation of the 5- $\mathrm{HT}_{3}$ and $\mathrm{nACh}$ receptors on the soma of the motor neuron caused the production of action potentials of $134 \mathrm{mV}$. Lotronex blocked the serotonergic pathway between the primary sensory and motor neurons. Thus under the assumption that there was no ACh in the system and neither nACh nor $\mu \mathrm{ACh}$ receptors were functional, Lotronex caused a disruption in the propagation of the wave of depolarization along the myenteric nervous plexus. The normal electrical signal transduction was made possible through the co-existing cholinergic mechanisms.

In contrast, activation of $5-\mathrm{HT}_{4}$ receptors changed the pattern of neuronal electrical activity into a long lasting excitatory mode. Action potentials of amplitude $114 \mathrm{mV}$ were produced. Concurrent stimulation of 5- $\mathrm{HT}_{4}$ and $\mathrm{nACh}$ receptors initiated strong sodium ion current and the generation of spikes of amplitude $116.5 \mathrm{mV}$.

\subsubsection{Smooth muscle syncytium}

In the absence of acetylcholine, the administration of Lotronex (GSK) (fig. 1) increased the frequency of slow wave electrical activity, hyperpolarized the smooth muscle syncytium and eliminated the generation of spikes on the crests of slow waves. As a result, there was a significant reduction in the force of contraction in the gut. Co-release of $\mathrm{ACh}$ into the system and a subsequent activation of the $\mathrm{nACh}$ and $\mu \mathrm{ACh}$ receptors restored the production of high amplitude action potentials of $57.4 \mathrm{mV}$, and caused long-lasting tonic type contractions (fig. 1,2). The latter impaired propulsive activity of the gut and the propulsion of the pellet. Only after the removal of ACh from the receptor sites the organ did resume its propulsive activity in the presence of (Lotronex).

\subsection{Effect of benzodiazepines}

Pharmacological and radiohistochemical studies have revealed two types of $\gamma$ aminobutyric acid (GABA) receptors in the gut: $\mathrm{GABA}_{\mathrm{A}}$ and $\mathrm{GABA}_{\mathrm{B}} \cdot \mathrm{GABA}_{\mathrm{A}}$ receptors are present on the somas of the primary sensory neurons and smooth muscle cells. They are coupled to ligand gated L-type $\mathrm{Ca}^{2+}$ and $\mathrm{Cl}^{-}$channels on smooth muscle [16]. In presence of benzodiazepines the primary sensory neuron generated an action potential of an average amplitude $81.3 \mathrm{mV}$. The pattern of 

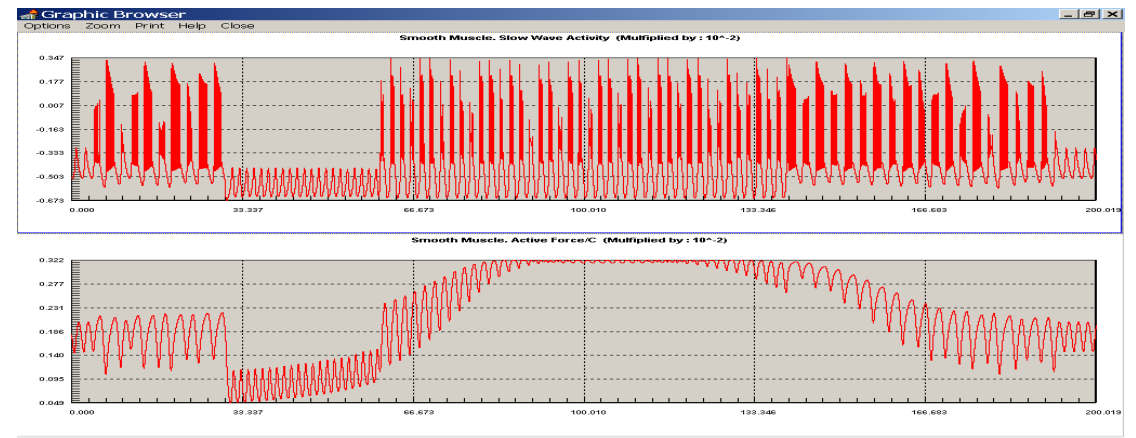

Figure 1: Effect of Lotronex (GSK) on myoelectrical (upper trace) and mechanical (lower trace) activities of the circular smooth muscle layer.
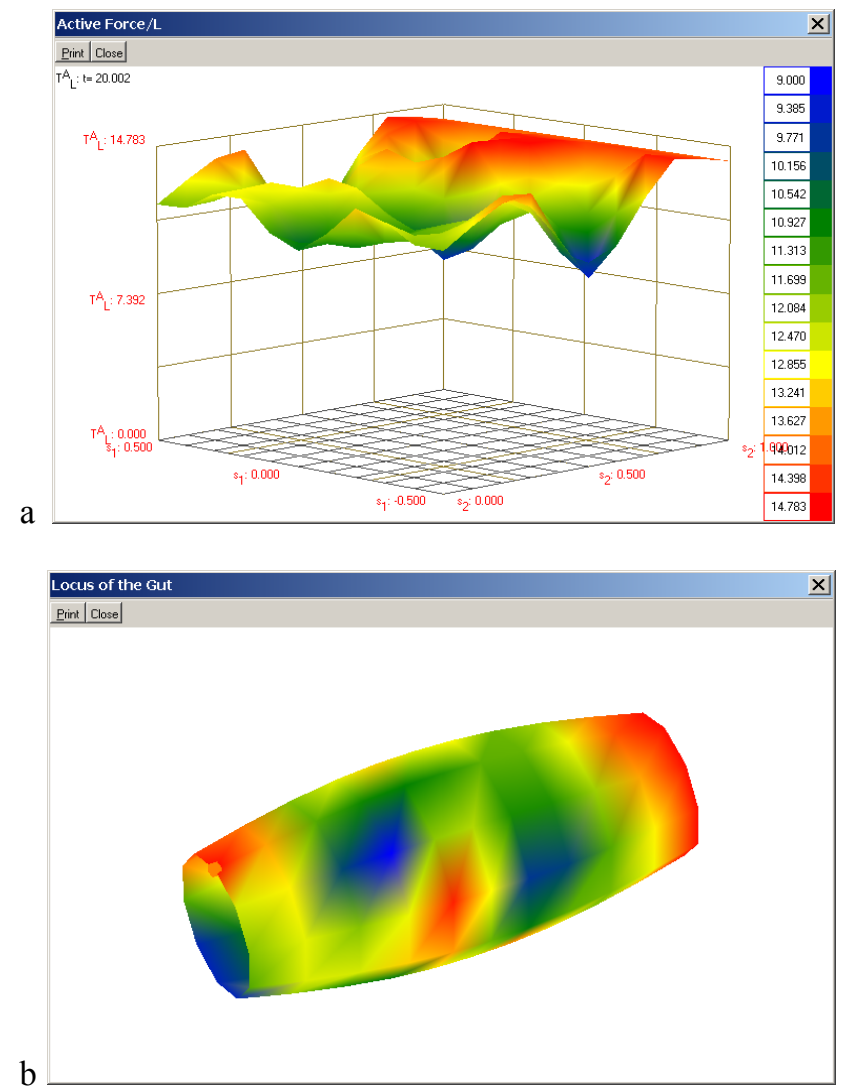

Figure 2: $\quad$ Effect of Lotronex (GSK). Active force distribution in the circular smooth muscle layer of the gut: plain (a) and 3D (b) presentations. 
discharge resembled that of the bursting chaos type of electrical activity with the frequency 8-10 (Hz). The smooth muscle syncytium was hyperpolarized that affected slow wave electrical activity. Small amplitude and reduced frequency oscillations in membrane potential were generated. They were insufficient to sustain influx of extracellular calcium into the cell and activate contractions. As a result, the syncytium remained in a steady, relaxed, and non-oscillatory state.

To alleviate the inhibitory effects of benzodiazepines on intestinal motility ryanodine was added into the system. Application of ryanodine did not change the dynamics of electrical activity of the primary sensory and motor neurons. Significant changes were seen, however, in the dynamics of the membrane currents in the smooth muscle syncytium. The intensity and the dynamics of activation-inactivation of inward and outward ion currents increased significantly. Thus ryanodine restored the slow wave myoelectrical activity of the smooth muscle, notably phase II of the migrating myoelectrical complex. The syncytium generated phasic contractions of a small strength, which were enough to sustain propulsive peristaltic activity of the gut in presence of benzodiazepines.

In in vitro experiments on cultured neurons and smooth muscle cells benzodiazepines at very low concentrations potentiate $\mathrm{GABA}_{\mathrm{A}}$ receptor-mediated $\mathrm{ACh}$ release. We studied the cooperative action of $\mathrm{ACh}$, erythromycin and ryanodine to correct the inhibitory effect of benzodiazepines on motility of the gut. The simultaneous application of these compounds had drastic effect on the smooth muscle, with little or no change in the neuronal elements. ACh with the other agents increased the intensity of all ionic membrane currents, with a rise in both their amplitudes and frequencies. The amplitude and frequency of slow wave increased and high amplitude action potentials, $61.4 \mathrm{mV}$ were generated on the crests of the slow waves resembling those observed during phases II and III of the migrating myoelectrical complex. As a result, the intestine produced intense contractions.

\section{Discussion}

A meticulous comparative analysis and validation of the simulation results with the in vitro and in vivo experimental data are given elsewhere $[13,15]$. Here, the focus is given to the aftermath of findings and how they affected the process of drug (Lotronex) development. The serotonergic signaling system was chosen as a target to treat hypersensitivity based on the fact that: $90 \%$ of all serotonin are stored in the gut, $5-\mathrm{HT}_{3}$ and $5-\mathrm{HT}_{4}$ type receptors are present on the neurons of the myenteric plexus and smooth muscle, and serotonin has secretory and prokinetic effects in the gastrointestinal tract. Electrophysiological studies on isolated single ion channels and cells showed that block of the 5- $\mathrm{HT}_{3}$ receptors had an inhibitory effect on their excitability. Our numerical results indicate that treatment of the primary and motor neurons with Lotronex in the absence of other neurotransmitters blocked the spread of the wave of depolarization in the afferent pathway of the gut. 
In vitro 5- $\mathrm{HT}_{3}$ receptor antagonists demonstrated inhibitory effect on propulsive activity of isolated segments of the colon. A note should be made that in some experiments anticholinergic drugs were added to avoid spontaneous mechanical activity of a specimen. Our numerical results concur with the recorded response of the gut to treatment with Lotronex alone.

Analysis of the above data within the old frame that the successive steps in the signal transduction and information decoding process are organized in a "linear and independent" manner, led drug developers to assume that a selective block of $5-\mathrm{HT}_{3}$ receptors in the human body should reduce hypersensitivity and motility of the gut. The designed drug (Lotronex), however, had dreadful effects on patients including ischemic colitis and severe constipation [17].

Trapped in the concept of "reductionism" - single channel function, recordings from isolated cultured cells, animal models, researchers neglected multilevel nonlinear interactions that inevitably occur in the human body. One of the possible mechanisms is the co-existence of multiple neurotransmitters and types of receptors that serve different metabolic pathways. Immunohistochemical staining of neurons of the myenteric plexus revealed co-localization by 5-HT and ACh [18]. However, the electropharmacological significance of the fact on co-transmission cannot be currently assessed experimentally.

The mathematical model and computer simulations provided a combined "reduction-integrated" representation of the sequence of events related to the dynamics of visceral perception at the protein (ion channel), cellular (the primary sensory and motor neurons), tissue (smooth muscle syncytia) and organ (a segment of the gut) levels. The results of numerical investigations showed that after a selective inhibition of $5-\mathrm{HT}_{3}$ receptors the existing cholinergic signaling pathway and unaffected $5-\mathrm{HT}_{4}$ receptors could serve as an adaptive mechanism to maintain the normal signal transduction. Surprisingly, their activation led to tonic type contractions, which could explain the slowdown in the pellet propulsion by the gut clinically, manifested as constipation.

There are more than 20 neurotransmitters and associated receptor types that are identified in the gut. Their integrated function can be only evaluated by the methods of computer simulation. For example, the excitatory amino acid signaling system engages glutamate and L-aspartate as neurotransmitters acting at $\mathrm{N}$-methyl $\mathrm{d}$-aspartate and $\alpha$-amino-3-hydroxy-5-methylisoxazole-4-propionic acid receptors, respectively. There is experimental evidence that glutamate and acetylcholine are co-stored in same vesicles at the presynaptic nerve terminal [19]. The excessive release of ACh and glutamate, as a compensatory measure to the inhibition of 5- $\mathrm{HT}_{3}$ receptors, may cause the overexcitation of neurons the phenomenon known as excitotoxicity, and their imminent death [14, 20]. Following integrated system biology approach in decision making would cause someone to anticipate potential side-effects like ischemic (gangrenous) colitis and constipation.

Combination therapy with various psychotropic agents, including benzodiazepines and barbiturates, can depress the motility of the gastrointestinal tract both in vivo and in vitro [21]. Results of our numerical experiments on the role of benzodiazepines displayed a series of intrinsic processes that are obscure 
in the usual biological preparations on the somas of neurons and in the muscle syncytium [22]. Only with the modeling approach it was possible to observe in parallel the changes in processes that contribute to the net effect - the cessation of contractions. This ability enabled us to assess pharmacological "interventions" in an effort to annul undesirable "side-effects". Thus, the addition of ryanodine restored the irregular pattern of phase II of the migrating myoelectrical complex and the simultaneous application of erythromycin, ryanodine, and ACh returned the gut to activity, which can be viewed as a clinically desirable correction.

Mathematical modeling and computer simulation of biological systems as an emerging technology offers a cheap, practical and biologically meaningful solution to the problem of expedient and safe drug design and development through its ability to explore a wide range of experimental conditions in a virtual environment. The "in silico" system biology approach allows:

i) expedient and rational identification and validation of drug targets against the background in which the function of a target is expressed;

ii) optimal therapeutic profiling of a new drug and systematic exploration of whether the most effective treatment is a drug that acts specifically on a single target or one that acts at multiple targets;

iii) analytical assessment of the effects of different classes of drugs and recommendation on modifications of the compounds to improve their efficacy;

iv) investigation of issues of clinical safety of a drug(s);

v) understanding of the impact of physiological variables on drug action in ways that cannot be adequately represented in even the most complex animal models and thus prediction of pharmacobiological effects of new compounds before they run expensive and timeconsuming in vivo and in vitro experiments.

However, most existing software is incapable of analyzing and integrating complex interactions of molecular, cellular, tissue, organ and system activities under conditions involving one or more lead compounds. The desired computational platforms should be based on new theoretical concepts of the physiology of biological systems and the pathogenesis of diseases. Till such concepts are established in medicine and biology models will remain unsatisfactory and the pharmaceutical industry will be skeptical in employing them.

In conclusion, drug developers, researchers and physicians using the mathematical modeling technology can speed up critical decisions concerning the rational direction of experimental work at different stages of a lengthy process of drug discovery and development and thus bring better, safer and more effective drugs to patients.

\section{References}

[1] Kenakin, T., New concepts in drug discovery: collateral efficacy and permissive antagonism. Nature Reviews. Drug Discovery, 4, pp. 919-927, 2005 
[2] Clayton, T.A., Lindon, J.C., Cloarec, O., Antti, H., Charuel, C., Hanton, G., Provost, J-P., Le Net, J-L., Baker, D., Walley, R.J., Everett, J.R. \& Nicholson, J.K., Pharmaco-metabonomic phenotyping and personalized drug treatment. Nature, 440, pp. 1073-1076, 2006

[3] Roses, A., Pharmacogenetics and drug development: the path to safer and more effective drugs. Nature Reviews. Genetics, 5, pp. 645-656, 2004

[4] Schneider, G. \& Fechner, U., Computer based de novo design of drug-like molecules. Nature Reviews. Drug Discovery, 4, pp. 649 - 663, 2005

[5] Newman M.E., Properties of single clustered networks. Phys. Rev. Ser. E, Stat. Nonlin. Soft Matter Phys., 68, pp. 261-272, 2003

[6] Apic, G., Ignjatovic, T., Boyer, S. \& Russell, R.B., Illuminating drug discovery with biological pathways. FEBS Letters, 579, pp. 1872-1877, 2005

[7] The Fruits of Genomics. Lehman Brothers. Press Release. Jan. 31, 2001. Online. http://www.lehman.com

[8] Frantz, S., Pharma faces major challenges after a year of failures and heated battles. Nature Reviews. Drug Discovery, 6, pp. 5-7, 2007

[9] Jonker, D.M., Visser, S.A.G., van der Graaf, P.H., Voskuyl, R.A. \& Danhof, M., Towards a mechanism-based analysis of pharmacodynamics drug-drug interactions in vivo. Pharmacology \& Therapeutics, 106, pp. 118,2005

[10] Stewart, K.D., Shiroda, M. \& Craig A.J., Drug GURU: a computer software program for drug design using medicinal chemistry rules. Bioorganic and Medicinal Chemistry, 14, pp. 701-7022, 2006

[11] Schoebert, B., Nielsen, U.B. \& Paxson, R., Model-based design approached in drug discovery: A parallel to traditional engineering approaches. IBM Journal of Research \& Development, 50(6), pp. 645651,2006

[12] Miftakhov, R. \& Fedotov, E.M., The concept of a functional unit of the gut. Advances in Fluid Mechanics $V$, A. Mendes, M. Rahman \& C. Brebbia (Eds.), WIT Press, Southampton, pp. 553-561, 2004

[13] Miftahof, R., Numerical simulation of the role of co-transmission by acetylcholine and serotonin on motility of the gut. Journal of Mechanics in Medicine and Biology, 6(4), pp. 399-428, 2006

[14] Miftahof, R. \&. Akhmadeev, N., Computer simulation of co-transmission by excitatory amino acids and acetylcholine in the enteric nervous system. Journal of Mechanics in Medicine and Biology, 2007 (in press)

[15] Miftahof, R. \&. Akhmadeev, N., Numerical simulation of effects of multiple neurotransmission on intestinal propulsion of a non-deformable bolus. Computational and Mathematical Methods in Medicine, 2007 (in press)

[16] Zeiter, D.K., Li, X. \& Broussard, D.L., Identification of the GABA $_{A}$ receptor $\alpha$-subunit mRNA in rat intestine. Molecular Brain Research. 39, pp. 241-244, 1996

[17] Irritable bowel drug Lotronex yanked by FDA, www.MedicineNet.com 
[18] Tack, J. \& Sarnelli, G., Serotonergic modulation of visceral sensation: upper gastrointestinal tract. Gut, 51, pp. i77-i80, 2002

[19] Kirchgessner, A.L., Glutamate in the enteric nervous system. Current Opinion in Pharmacology, 1, pp. 591-596, 2001

[20] Kirchgessner, A.L., Liu, M.T. \& Alcantara, F., Excitotoxicity in the enteric nervous system. Journal of Neuroscience, 17, pp. 8804-8816, 1997

[21] Christensen, J., Dent, J.R., Malagelada, J. R. \& Wingate, D.L., Pseudoobstruction. Gastroenterology International. 3, pp. 107-119, 1990

[22] Miftakhov, R.N., Abdusheva, G.R. \& Christensen, J., Numerical simulation of motility patterns of the small bowel. II. Comparative pharmacological validation of a mathematical model. Journal of Theoretical Biology. 200, pp. 261-290, 1999 